\title{
Surgical Therapy of Cholangiocarcinoma
}

\author{
Arnold Radtke Alfred Königsrainer \\ Department of General, Visceral and Transplant Surgery, Comprehensive Cancer Center, University of Tübingen, Tübingen, Germany
}

\section{Keywords}

Cholangiocarcinoma - Bile duct carcinoma ·

Surgical strategy · Hepatectomy · Liver transplantation . Portal vein embolization - ALPPS

\section{Summary}

Background: The majority of patients with cholangiocarcinoma present with advanced disease that is often challenging to diagnose and to treat. The optimal preoperative evaluation requires a coordinated multidisciplinary approach. Surgical resection is the mainstay of therapy. Methods: This systematic review delineates surgical treatment strategies for cholangiocarcinoma in general as well as special considerations concerning the particular tumor localization. A literature search (see keywords) was conducted using PubMed and publications between 1990 and 2016 regarding resectable and advanced cholangiocarcinoma were reviewed. Selected studies were utilized based on their significance and innovation. Results: The type and extent of resection performed depends on the location of the cholangiocarcinoma within the liver or biliary tree and the extent of local tumor invasion. The common surgical strategy contains: (i) for intrahepatic tumors: tailored partial hepatectomy combined with extended hilar, suprapancreatic, celiac axis lymphadenectomy, (ii) for hilar tumors: complete resection of the extrahepatic biliary tree combined with extended hepatectomy inclusive of segment I, resection of portal vein bifurcation, and systematic N1/N2 lymphadenectomy, and (iii) for distal tumors: en bloc pancreatoduodenectomy combined with complete resection of the extrahepatic bile duct below the hepatic confluence and systematic N1/N2 lymphadenectomy. Pathologic confirmation is not required prior to resection. Preoperative biliary drainage and remnant liver volume augmentation are necessary in selected patients with intrahepatic or hilar cholangiocarcinoma considered for extensive liver resection. Conclusion: Cure for cholangiocarcinoma requires complete surgical resection with histologically negative margins. $\mathrm{R} 0$ resection provides a satisfactory long-term outcome in patients with lymph node-negative stage. Neoadjuvant treatment followed by liver transplantation provides long-term survival in highly selected cases with localized, unresectable, lymph nodenegative hilar cholangiocarcinoma.

(c) 2016 S. Karger GmbH, Freiburg

\section{Introduction}

Despite significant advances in systemic therapy only complete radical surgery provides a curative treatment option for patients with bile duct cancer [1].

Cholangiocarcinomas are known to potentially invade any part of the biliary tree and are categorized as intrahepatic and proximal or distal extrahepatic depending on their localization with respect to anatomical landmarks including second-order bile ducts, bile duct confluence, and cystic duct levels.

Several risk factors predisposing to cholangiocarcinoma associated with chronic inflammation of the biliary epithelium have been identified. Particularly in patients with primary sclerosing cholangitis (PSC) as well as in individuals with hepatolithiasis, bile stasis and recurrent bouts of subclinical cholangitis may contribute to carcinogenesis [2]. Other risk factors include parasitic infections, congenital biliary tree anomalies (e.g. bile duct cysts, Caroli's disease), viral hepatitis, and cirrhosis.

Cholangiocarcinoma originates from the intra- or extrahepatic bile duct epithelium. The vast majority are adenocarcinomas arising from periductal glands, whereas remaining histologic variants comprise $<5 \%$ of cases [3].

The morphology-based classification distinguishes three categories, i.e. periductal infiltrating, intraductal infiltrating, and massforming tumors [4]. The particular growth pattern of cholangiocarcinoma is characterized by infiltration and fibrosis of periductal tissues resulting in annular bile duct wall thickening, while poly-

\section{KARGER}

(๑) 2016 S. Karger GmbH, Freiburg

Fax +497614520714 
poid or nodular tumor growth appear extremely rarely [5]. The microscopic findings including vertical intraductal and lateral periductal infiltration, neurovascular invasion, and surrounding intraepithelial lesions have a decisive prognostic significance.

The macro- and microscopic features of cholangiocarcinoma explain the extreme limitations in detecting longitudinal tumor extent along the bile ducts and also radial spread into adjacent structures by preoperative radiological and endoscopic imaging [6]. Furthermore, in patients with biliary stents, local artifacts and secondary inflammatory changes can additionally limit the diagnostic accuracy of imaging.

The localization of the tumor is essential for the surgical strategy. In this regard, distal cholangiocarcinoma has the highest resectability and the most favorable outcome due to its anatomical properties when compared with the other tumor localizations [7]. Thus, anatomical localization and special growth pattern are essential for planning surgery.

In addition to macro- and microscopic features, the locoregional and distant lymph node involvement bears a significant prognostic value. However, the capacity for detecting lymph node metastases by high-quality cross-sectional imaging (magnetic resonance imaging, computed tomography) or endoscopic ultrasound is still unsatisfactory because of the poor correlation between lymph node size and their positivity for malignancy [8-9]. In that respect, fluorodeoxyglucose positron emission tomography was found to provide a much higher specificity for detecting malignant lymphadenopathy [10].

Consequently, patients with potentially operable cholangiocarcinoma should always be planned for surgical exploration by laparoscopy or laparotomy inclusive of histological assessment. In the current opinion, the evidence of peritoneal carcinomatosis indeed precludes extended resection while the presence of technically resectable liver metastases does not definitely contraindicate radical surgery [1].

Numerous studies report that aggressive surgical strategies combined with multimodal treatment concepts allowed for the obvious increase of curative resections $[1,11]$.

Although recent advances in surgical techniques and perioperative management have offered increased resectability and improved surgical outcomes, unfortunately more than half of the operated patients even following curative resection exhibit a recurrent disease, and their overall long-term outcome remains unsatisfactory [12].

These patients benefit slightly from chemoradiotherapy but are in selected cases suitable for redo resection with curative intent [13].

The role of adjuvant chemo- and radiotherapy strategies to provide a better local disease control especially in low-risk patients is not satisfactorily cleared yet. The current practice recommends adjuvant therapies in patients with positive nodes or resection margins (R1). Particularly in patients at high risk of recurrence their benefit seems to be clear [14].

\section{Surgical Strategy and Technique - General Considerations}

In general, surgical treatment for cholangiocarcinoma is determined by the patient's clinical condition, functional liver volume specifically disturbed by severe cholestasis, local extent of the tumor including vascular and/or parenchymal involvement, as well as absence or presence of metastatic disease.

The complete surgical en bloc resection with negative macroscopic and microscopic margins (R0 resection) as well as lymph node metastases are decisive criteria regarding long-term survival in patients with cholangiocarcinoma $[11,15,16]$.

Irrespective of the tumor localization, extensive liver resection is nearly always required to achieve R0 resection [17].

In patients considered for extended hepatectomy, preoperative portal vein embolization is mainly necessary to induce compensatory hypertrophy of the remnant liver [18]. Consequently, in those particular patients a preoperative biliary drainage via endoscopic retrograde cholangiopancreatography or percutaneous transhepatic cholangiography drainage is commonly indicated to relieve the intrahepatic cholestasis [19].

Biliary drainage is also clearly indicated in septic patients due to congestive cholangitis, likewise in the evidence of severe malnutrition or hepatic insufficiency [20,21].

\section{Intrahepatic Cholangiocarcinoma}

$5-10 \%$ of cholangiocarcinomas arise from peripheral bile ducts within the liver parenchyma distal to the second-order bile ducts [3].

The common strategy containing major hepatectomy combined with extended lymphadenectomy involving hilar, suprapancreatic as well as retroperitoneal compartments allows for a complete surgical en bloc resection with negative macroscopic and microscopic margins (R0 resection) in less than $50 \%$ of patients despite the preference (60\%) for extended hepatectomies ( $>4$ segments) [22].

Periductal tumor growth along the periportal fields resulting in extensive infiltration of surrounding tissue seems to be one of the explanations for the unsatisfactory outcome [23].

Moreover, the presumed beneficial effect of systematic lymphadenectomy is still not satisfactorily proven [22]. Although positive lymph nodes are a significant prognostic factor, aggressive lymphadenectomy is being controversially discussed. The opponents argue that the high biliary morbidity due to ischemic bile duct injuries may offset the potential oncological benefit, and thus some centers limit the indication to only centrally localized tumors [23].

Our own policy recommends a priori systematic lymphadenectomy with extended hepatectomy regardless of peripheral or central tumor localization. However, a detailed surgical exploration including histological assessment of corresponding lymph node compartments precedes our decision making in each patient.

While positive hilar or suprapancreatic nodes (N1) do not contraindicate surgery, we have strong reservations to go along with surgery in the evidence of malignant retroperitoneal (N2) lymphad- 
enopathy, strictly limiting the indication to only those resectable patients who are symptomatic due to the tumor disease. Our current practice considers patients with positive retroperitoneal nodes for palliative chemotherapy, with the option of secondary surgery with curative intent if a satisfactory response has been achieved.

In our series, nearly $80 \%$ of patients underwent R 0 resection, and in single cases with advanced disease a complete radical resection following primary chemotherapy was possible.

\section{Extrahepatic Distal Cholangiocarcinoma}

In $30-40 \%$ of cases cholangiocarcinoma involves the middle and distal common bile duct arising below the cystic duct level [3].

The common surgical strategy consists of en bloc pancreatoduodenectomy (Whipple operation, optionally pylorus-preserving Longmire-Traverso procedure), combined with complete resection of the extrahepatic bile duct below the hepatic confluence and systematic N1/N2 lymphadenectomy.

Long-term survival rates over $50 \%$ in lymph node-negative stage followed by R0 resection have been widely reported [24].

However, more extensive surgery is required to provide negative margins (R0 resection) when intrahilar tumor invasion exceeded the biliary confluence level. In such cases, extended bile duct resection inclusive of biliary confluence and segment I or combined Whipple operation and major hepatecomy are possible curative options in highly selected patients [25].

Unfortunately, hepato-pancreatoduodenectomy bears a substantial operative risk, with mortality rates of up to $10 \%$ in experienced hands, when compared with standard Whipple operation (5\%) [26].

Given the fact that more than $40 \%$ of severe complications af fect the pancreatic remnant (i.e. bleeding, pancreatitis, anastomotic leak) being triggered by the underlying liver dysfunction, some centers favor a stepwise procedure considering liver resection after Whipple operation [25].

Thus, thoughtful planning of surgery is always necessary to precisely determine the operative limits and the most optimal strategy (i.e. preoperative portal vein embolization) for each individual patient.

There is common consensus that preoperative biliary drainage is not required unless additional liver resection or neoadjuvant therapy is planned [7].

\section{Extrahepatic Proximal, (Peri-)Hilar \\ Cholangiocarcinoma - ‘Klatskin’ Tumor}

The vast majority (60-70\%) of cholangiocarcinomas involve the bile duct confluence arising distal to the cystic duct level [3]. They are further classified into four main categories according to the Bismuth-Corlette classification [27]:

- Type I: tumors involving the common hepatic duct below the biliary confluence;
Table 1. Preoperative resectability assessment of hilar cholangiocarcinoma a modified clinical tumor staging system of Blumgart [28]

\section{Criteria of unresectability}

Extrahepatic metastases / non-resectable liver metastases

Bilateral tumor extension involving the second-order bile ducts

Total portal vein occlusion

Tumor invasion into second-order bile ducts with contralateral portal vein thrombosis

Temporary contraindication to surgery

Inadequate remnant liver volume

Liver volume augmentation by portal vein embolization

Extensive cholestasis / biliary sepsis

Biliary drainage by ERCP / PTCD, antibiotics

ERCP = Endoscopic retrograde cholangiopancreatography;

$\mathrm{PTCD}=$ percutaneous transhepatic cholangiography drainage.

- Type II: tumors involving the biliary confluence;

- Type IIIa: tumors involving the biliary confluence extending into the right hepatic duct;

- Type IIIb: tumors involving the biliary confluence extending into the left hepatic duct;

- Type IV: tumors involving the biliary confluence extending into both right and left hepatic duct or multifocal tumors.

Paying heed to the close correlation between tumor localization within the biliary confluence and portal vein infiltration or hepatic metastases, the Bismuth-Corlette classification proved to be helpful in planning surgery for radical tumor resection.

Decisive for the proper indication and patient selection during preoperative workup are systematic evaluation of the local extent of the tumor, detection of intrahepatic or distant metastases, estimation of the future liver remnant volume, and alleviation of severe cholestasis.

However, in most cases the preoperative staging is seriously limited, making a reliable assessment of the three-dimensional tumor extent practically impossible.

The overall resectability rate of patients with hilar cholangiocarcinoma reported in the literature is approximately $65 \%$ [1].

The main reason for unresectability at exploration is underestimation of local tumor extent on preoperative imaging, followed by extensive vascular involvement and occult metastatic disease [28].

The tumor staging system of Blumgart recently modified by the Sloan-Kettering group takes into consideration the drawbacks of preoperative imaging and has the potential to usefully assist in patient selection for major resections (table 1).

Finally, the type and extent of intended resection depends on the tumor location within the biliary confluence and the depth of tumor invasion inside the liver (longitudinal extent) and likewise on the degree of local invasion into the adjacent periductal structures including the infiltration of portal vein and hepatic arteries (radial extent).

Our own experience, as confirmed by others, showed R0 resection rates of less than $20 \%$ following isolated resection of the extrahepatic bile duct. For that reason, limited resection has been restricted to only Bismuth-Corlette type I hilar cholangiocarcinoma [1]. 
In contrast, complete $\mathrm{R} 0$ resection for Bismuth-Corlette types II-IV hilar cholangiocarcinoma involves extensive resection of both the extra- and intrahepatic biliary tree, requiring removal of the ipsilateral hemiliver (mostly extended hepatectomy), en bloc resection of portal vein bifurcation, and systematic lymphadenectomy involving $\mathrm{N} 1$ and $\mathrm{N} 2$ compartments $[1,21]$.

The aggressive strategy presented by the Nagoya group further improved long-term outcomes and could be finally established as a state-of-the-art operation for Bismuth-Corlette types II-IV hilar cholangiocarcinoma [19]. In experienced centers, R0 resection rates of up to $75 \%$ and 5 -year survivals of more than $60 \%$ in nodenegative stage were reported [1].

This standardized operation combines complete resection of the extrahepatic duct proximally limited by the upper duodenal edge with right trisectionectomy (resection of segments 5-8+4) inclusive of segment I, en bloc resection of portal vein bifurcation followed by end-to-end reconstruction between the portal vein trunk and left portal vein, and systematic N1/N2 lymphadenectomy [1].

Combined vascular resection involving the portal vein and hepatic artery in advanced hilar cholangiocarcinoma is technically feasible and can be a surgical option for highly selected patients [29].

The rationale to favor right trisectionectomy in hilar cholangiocarcinoma is based on anatomical considerations, depicting several properties that facilitate parenchyma transection and surgical dissection in the left lateral lobe [30]:

(1) Separate left hepatic vein providing independent venous drainage of segments 2 and 3.

(2) Umbilical plate ('own hilus') containing separate bile duct and portal vein confluence (Rex sinus).

(3) Left hepatic duct of long extrahepatic segment well accessible for surgical assessment.

The preferential en bloc resection of the portal vein bifurcation is reasonable because of the distinct anatomical proximity to the central bile duct and the biliary confluence. Furthermore, inclusion of segment I into the resection specimen is beneficial because cholangiocarcinoma can extend into the caudate lobe via small caudal branches draining into the right and left hepatic duct and into the biliary confluence itself, resulting in common caudal lobe involvement, as seen in $43-100 \%$ of patients [31].

Patients who are considered for extended hepatectomy with an estimated remnant liver volume $<25-30 \%$ require volume augmentation by preoperative ipsilateral portal vein embolization in order to avoid postoperative liver failure due to a small-for-size situation [32]. When right trisectionectomy is intended, right portal vein embolization including segment 4 branches is required to induce compensatory hypertrophy of left lateral segments $2 / 3$. In selected cases, additional embolization of the ipsilateral hepatic vein as described by the Seoul and Calgary groups effectively intensified the compensatory parenchyma growth in the future liver remnant [33,34].
In the setting of left-sided cholangiocarcinoma (Bismuth-Corlette types IIIa and IV), extended left trisectionectomy (segments 1-4, 5-8) following left portal vein embolization may be the preferable option for the patient. Additional multiductal biliary reconstruction is likely to be necessary after this complex resection [35].

In contrast, a two-step resection with in situ liver split and right portal vein ligation according to the ALPPS (i.e. 'Associating Liver Partition and Portal Vein Ligation for Staged Hepatectomy') concept is not recommended in functionally deranged and cholestatic livers because of the extremely high risk for morbidity and mortality [36].

Although surgical resection remains the standard of care for hilar cholangiocarcinoma, some selected patients with technically unresectable lymph node-negative tumors can benefit from total hepatectomy combined with complete extrahepatic bile duct resection followed by liver transplantation [37].

A trimodal neoadjuvant protocol incorporating external beam and endoluminal radiation combined with systemic chemotherapy initially proposed by the Mayo group showed a promising longterm survival of $59 \%$ [38].

There is a lack of reported randomized trials supporting a routine use of adjuvant chemoradiotherapy after curative resection for hilar cholangiocarcinoma. However, there are convincing data to suggest that adjuvant chemoradiotherapy may be beneficial, particularly for lymph node-positive and bile duct margin-positive (R1) resections [39].

\section{Conclusion}

Cholangiocarcinoma appears extremely challenging to diagnose and treat independent of the anatomic location. The plausible explanation includes the particular peri-/intraductal growth pattern accompanied by fibrosis of periductal tissues.

Radical surgical treatment still remains the only curative option with the potential of complete tumor healing.

Besides the primary tumor stage being an independent fateful variable, the avoidance of incomplete surgical resection with micro- or macroscopic positive margins (R1/R2 resection) is seminal for the long-term outcome in those patients.

Evaluation by an experienced hepatobiliary surgeon is therefore recommended wherever cholangiocarcinoma is suspected, ideally before biliary stenting potentially complicates the subsequent tumor staging and surgery due to secondary biliary inflammation or infection.

\section{Disclosure Statement}

The authors disclose no potential conflicts of interest and no external funding sources. 


\section{References}

1 Nagino M, Ebata T, Yokoyama Y, Igami T, Sugawara G, Takahashi Y, Nimura Y: Evolution of surgical treatment for perihilar cholangiocarcinoma. Ann Surg 2013; 258:129-140.

2 Patel T: Cholangiocarcinoma. Nat Clin Pract Gastroenterol Hepatol 2006;3:33-42.

3 Cidon EU: Resectable cholangiocarcinoma: reviewing the role of adjuvant strategies. Clin Med Insights Oncol 2016;10:43-48.

4 Yamasaki S: Intrahepatic cholangiocarcinoma: macroscopic type and stage classification. J Hepatobiliary Pancreat Surg 2003;10:288-291.

5 Lim JH, Park CK: Pathology of cholangiocarcinoma. Abdom Imaging 2004;29:540-547.

6 Okumoto T, Sato A, Yamada T, Takase K, Matsuhash T, Tsuda M, Seiji K, Ishibashi T, Higano S, Katayose Y, Unno M, Takahashi S: Correct diagnosis of vascular encasement and longitudinal extension of hilar cholangiocarcinoma by four-channel multidetector-row computed tomography. J Exp Med 2009;217:1-8.

7 Esnaola NF, Meyer JE, Karachristos A, Maranki JL, Camp ER, Denlinger CS: Evaluation and management of intrahepatic and extrahepatic cholangiocarcinoma. Cancer 2016;122:1349-1369.

8 Manfredi R, Barbaro B, Masselli G, Vecchioli A, Marano $P$ : Magnetic resonance imaging of cholangiocarcinoma. Semin Liver Dis 2004;24:155-164.

$\checkmark$ Cho ES, Park MS, Yu JS, Kim MJ, Kim KW: Biliary ductal involvement of hilar cholangiocarcinoma: multidetector computed tomography versus magnetic resonance cholangiography. J Comput Assist Tomogr 2007;31:72-78.

10 Kato T, Tsukamoto E, Kuge Y, Katoh C, Nambu T, Nobuta A, Kondo S, Asaka M, Tamaki N: Clinical role of ${ }^{18}$ F-FDG PET for initial staging of patients with extrahepatic bile duct cancer. Eur J Nucl Med Mol Imaging 2002;29:1047-1054.

11 Endo I, Gonen M, Yopp AC, Dalal KM, Zhou Q, Klimstra D, D’Angelica M, DeMatteo RP, Fong Y, Schwartz L, Kemeny N, O’Reilly E, Abou-Alfa GK, Shimada H, Blumgart LH, Jarnagin WR: Intrahepatic cholangiocarcinoma: rising frequency, improved survival, and determinants of outcome after resection. Ann Surg 2008;248:84-96.

12 Jung SJ, Woo SM, Park HK, Lee WJ, Han MA, Han SS, Kim SH, Park SJ, Kim TH, Koh YH, Hong EK: Patterns of initial disease recurrence after resection of biliary tract cancer. Oncology 2012;83:83-90.

13 Takahashi Y, Ebata T, Yokoyama Y, Igami T, Sugawara G, Mizuno T, Nimura Y, Nagino M: Surgery for recurrent biliary tract cancer: a single-center experience with 74 consecutive resections. Ann Surg 2015;262: 121-129.

14 Im JH, Seong J, Lee IJ, Park JS, Yoon DS, Kim KS, Lee WJ, Park KR: Surgery alone versus surgery followed by chemotherapy and radiotherapy in resected extrahepatic bile duct cancer: treatment outcome analysis of 336 patients. Cancer Res Treat 2016;48:583-595.
Khan SA, Davidson BR, Goldin RD, Heaton N, Karani J, Pereira SP, Rosenberg WM, Tait P, Taylor-Robinson $\mathrm{SD}$, Thillainayagam AV, Thomas $\mathrm{HC}$, Wasan $\mathrm{H}$ : Guidelines for the diagnosis and treatment of cholangiocarcinoma: an update. Gut 2012;61:1657-1669.

16 Kitagawa Y, Nagino N, Kamiya J, Uesaka K, Sano T, Yamamoto H, Hayakawa N, Nimura Y: Lymph node metastasis from hilar cholangiocarcinoma: audit of 110 patients who underwent regional and paraaortic node dissection. Ann Surg 2001;233:385-392.

17 Neuhaus P, Jonas S, Settmacher U, Thelen A, Benckert C, Lopez-Haenninen E, Hintze RE: Surgical treatment of proximal bile duct cancer: extended right lobe resection increases resectability and radicality. Langenbecks Arch Surg 2003;388:194-200.

18 Higuchi R, Yamamoto M: Indications for portal vein embolization in perihilar cholangiocarcinoma. J Hepatobiliary Pancreat Sci 2014;21:542-549.

19 Nimura Y, Kamiya J, Kondo S, Nagino M, Uesaka K, Oda K, Sano T, Yamamoto H, Hayakawa N: Aggressive preoperative management and extended surgery for hilar cholangiocarcinoma: Nagoya experience. J Hepatobiliary Pancreat Surg 2000;7:155-162.

20 Anderson CD, Pinson CW, Berlin J, Chari RS: Diagnosis and treatment of cholangiocarcinoma. Oncologist 2004;9:43-57.

21 Mansour JC, Aloia TA, Crane CH, Heimbach JK, Nagino M, Vauthey JN: Hilar cholangiocarcinoma: expert consensus statement. HPB (Oxford) 2015;17:691-699.

22 Simo KA, Halpin LE, McBrier NM, Hessey JA, Baker E, Ross S, Swan RZ, Iannitti DA, Martinie JB: Multimodality treatment of intrahepatic cholangiocarcinoma: a review. J Surg Oncol 2016;113:62-83.

23 Vitale A, Moustafa M, Spolverato G, Gani F, Cillo U, Pawlik TM: Defining the possible therapeutic benefit of lymphadenectomy among patients undergoing hepatic resection for intrahepatic cholangiocarcinoma. J Surg Oncol 2016;113:685-691.

24 Kim YS, Hwang IG, Park SE, Go SI, Kang JH, Park I, Oh SY, Ji JH, Song HN, Park SH, Kim ST, Park JO: Role of adjuvant therapy after R0 resection for patients with distal cholangiocarcinoma. Cancer Chemother Pharmacol 2016;77:979-985.

25 Ebata T, Yokoyama Y, Igami T, Sugawara G, Takahashi Y, Nimura Y, Nagino M: Hepatopancreatoduodenectomy for cholangiocarcinoma: a single-center review of 85 consecutive patients. Ann Surg 2012;256: 297-305.

26 Addeo P, Oussoultzoglou E, Fuchshuber P, Rosso E, Nobili C, Langella S, Jaeck D, Bachellier P: Safety and outcome of combined liver and pancreatic resections. Br J Surg 2014;101:693-700.

27 Bismuth H, Nakache R, Diamond T: Management strategies in resection for hilar cholangiocarcinoma. Ann Surg 1992;215:31-38.
28 Matsuo K, Rocha FG, Ito K, D’Angelica MI, Allen PJ, Fong Y, DeMatteo RP, Gonen M, Endo I, Jarnagin WR: The Blumgart preoperative staging system for hilar cholangiocarcinoma: analysis of resectability and outcomes in 380 patients. J Am Coll Surg 2012;215: 343-355.

29 Noji T, Tsuchikawa T, Okamura K, Tanaka K, Nakanishi Y, Asano T, Nakamura T, Shichinohe T, Hirano S: Concomitant hepatic artery resection for advanced perihilar cholangiocarcinoma: a case-control study with propensity score matching. J Hepatobiliary Pancreat Sci 2016;23:442-448.

30 Hirose T, Igami T, Ebata T, Yokoyama Y, Sugawara G, Mizuno T, Mori K, Ando M, Nagino M: Surgical and radiological studies on the length of the hepatic ducts. World J Surg 2015;39:2983-2989.

31 Nimura Y, Hayakawa N, Kamiya J, Kondo S, Shionoya S: Hepatic segmentectomy with caudate lobe resection for bile duct carcinoma of the hepatic hilus. World J Surg 1990;14:535-543.

32 Mise Y, Passot G, Wang X, Chen HC, Wei S, Brudvik KW, Aloia TA, Conrad C, Huang SY, Vauthey JN: A nomogram to predict hypertrophy of liver segments 2 and 3 after right portal vein embolization. J Gastrointest Surg 2016;20:1317-1323.

33 Hwang S, Ha TY, Ko GY, Kwon DI, Song GW, Jung DH, Kim MH, Lee SK, Lee SG: Preoperative sequential portal and hepatic vein embolization in patients with hepatobiliary malignancy. World J Surg 2015;39:29902998.

34 Munene G, Parker RD, Larrigan J, Wong J, Sutherland F, Dixon E: Sequential preoperative hepatic vein embolization after portal vein embolization for extended left hepatectomy in colorectal liver metastases. World J Surg Oncol 2013;11:134.

35 Uesaka K: Left hepatectomy or left trisectionectomy with resection of the caudate lobe and extrahepatic bile duct for hilar cholangiocarcinoma. J Hepatobiliary Pancreat Sci 2012;19:195-202.

36 Vennarecci G, Grazi GL, Sperduti I, Busi Rizzi E, Felli E, Antonini M, D'Offizi G, Ettorre GM: ALPPS for primary and secondary liver tumors. Int J Surg 2016;30: 38-44.

37 Marchan EM, Landry JC: Neoadjuvant chemoradiation followed by orthotopic liver transplantation in cholangiocarcinomas: the emory experience. J Gastrointest Oncol 2016;7:248-254.

38 Croome KP, Rosen CB, Heimbach JK, Nagorney DM: Is liver transplantation appropriate for patients with potentially resectable de novo hilar cholangiocarcinoma? J Am Coll Surg 2015;221:130-139.

39 Horgan AM, Amir E, Walter T, Knox JJ: Adjuvant therapy in the treatment of biliary tract cancer: a systematic review and meta-analysis. J Clin Oncol 2012; 30:1934-1940 